\title{
Utilizing a Sleep Mask to Reduce Patient Anxiety During Nail Surgery
}

\author{
Jose W. Ricardo, MD; Shari R. Lipner, MD, PhD
}

Patients undergoing nail surgery frequently experience anxiety. Nail procedures generally are performed under local anesthesia; common concerns include the sight of blood, the procedure itself, proceduralassociated disfigurement, and pain. Wearing a sleep mask diverts attention from the surgical field, thus reducing perioperative anxiety. Sleep masks are widely available and inexpensive.

Cutis. 2021;108:36.

\section{Practice Gap}

Perioperative anxiety is common in patients undergoing nail surgery. Patients might worry about seeing blood; about the procedure itself, including nail avulsion; and about associated pain and disfigurement. Nail surgery causes a high level of anxiety that correlates positively with postoperative pain ${ }^{1}$ and overall patient dissatisfaction. Furthermore, surgery-related anxiety is a predictor of increased postoperative analgesic use $^{2}$ and delayed recovery. ${ }^{3}$

Therefore, implementing strategies that reduce perioperative anxiety may help minimize postoperative pain. Squeezing a stress ball, hand-holding, virtual reality, and music are tools that have been studied to reduce anxiety in the context of Mohs micrographic surgery; these strategies have not been studied for nail surgery.

\section{The Technique}

Using a sleep mask is a practical solution to reduce patient anxiety during nail surgery. A minority of patients will choose to watch their surgical procedure; most become unnerved observing their nail surgery. Using a sleep mask diverts visual attention from the surgical field without physically interfering with the nail surgeon. Utilizing a sleep mask is cost-effective, with disposable sleep masks available online for less than $\$ 0.30$ each. Patients can bring their own mask, or a mask can be offered prior to surgery.

If desired, patients are instructed to wear the sleep mask during the entirety of the procedure, starting from anesthetic infiltration until wound closure and dressing application. Any adjustments can be made with the patient's free hand. The sleep mask can be offered to patients of all ages undergoing nail surgery under local anesthesia, except babies and young children, who require general anesthesia.

\section{Practical Implications}

Distraction is an important strategy to reduce anxiety and pain in patients undergoing surgical procedures. In an observational study of 3087 surgical patients, $36 \%$ reported that self-distraction was the most helpful strategy for coping with preoperative anxiety. ${ }^{4}$ In a randomized, open-label clinical trial of 72 patients undergoing peripheral venous catheterization, asking the patients simple questions during the procedure was more effective than local anesthesia in reducing the perception of pain. ${ }^{5}$

It is crucial to implement strategies to reduce anxiety in patients undergoing nail surgery. Using a sleep mask impedes direct visualization of the surgical field, thus distracting the patient's sight and attention from the procedure. Furthermore, this technique is safe and cost-effective.

Controlled clinical trials are necessary to assess the efficacy of this method in reducing nail surgery-related anxiety in comparison to other techniques.

\section{REFERENCES}

1. Navarro-Gastón D, Munuera-Martínez PV. Prevalence of preoperative anxiety and its relationship with postoperative pain in foot nail surgery: a cross-sectional study. Int J Environ Res Public Health. 2020;17:4481. doi:10.3390/ijerph17124481

2. Ip HYV, Abrishami A, Peng PWH, et al. Predictors of postoperative pain and analgesic consumption: a qualitative systematic review. Anesthesiology. 2009;111:657-677. doi:10.1097/ALN.0b013e3181aae87a

3. Mavros MN, Athanasiou S, Gkegkes ID, et al. Do psychological variables affect early surgical recovery? PLoS One. 2011;6:E20306. doi:10.1371/journal.pone.0020306

4. Aust H, Rüsch D, Schuster M, et al. Coping strategies in anxious surgical patients. BMC Health Serv Res. 2016;16:250. doi:10.1186/s12913-016-1492-5

5. Balanyuk I, Ledonne G, Provenzano M, et al. Distraction technique for pain reduction in peripheral venous catheterization: randomized, controlled trial. Acta Biomed. 2018;89(suppl 4):55-63. doi:10.23750 /abmv89i4-S.7115

From the Department of Dermatology, Weill Cornell Medicine, New York, New York.

The authors report no conflict of interest.

Correspondence: Shari R. Lipner, MD, PhD, 1305 York Ave, New York, NY 10021 (shl9032@med.cornell.edu).

doi: $10.12788 /$ cutis.0285 